\title{
Laparoscopic transhiatal surgery for an epiphrenic esophageal diverticulum derived from a jackhammer esophagus: a case report
}

\author{
Atsuro Fujinaga ${ }^{1 *}$, Tomotaka Shibata ${ }^{1}$, Tsuyoshi Etoh ${ }^{1}$, Kazuhiro Tada ${ }^{1}$, Kosuke Suzuki $^{1}$, Kohei Nishiki ${ }^{2}$, \\ Katsuhiro Ogawa', Yohei Kono ${ }^{1}$, Takahiro Hiratsuka', Tomonori Akagi ${ }^{1}$, Yoshitake Ueda ${ }^{3}$, Manabu Toujigamori ${ }^{1}$, \\ Hidefumi Shiroshita ${ }^{1}$, Norio Shiraishi ${ }^{3}$ and Masafumi Inomata ${ }^{1}$
}

\begin{abstract}
Background: An esophageal diverticulum is rare and is frequently associated with esophageal motility disorders. Jackhammer esophagus is also rare, is characterized by esophageal hypercontraction, and comprises $4.1 \%$ of esophageal motility disorders. Here, we report a case of a patient successfully treated by laparoscopic transhiatal surgery for an epiphrenic esophageal diverticulum derived from a jackhammer esophagus diagnosed with highresolution manometry (HRM).
\end{abstract}

Case presentation: The patient was a 78-year-old man who presented to the hospital with dysphagia. A diverticulum was detected in the lower part of his esophagus by upper gastrointestinal endoscopy. HRM was performed to investigate esophageal motility disorders. His integrated relaxation pressure was normal at $25.9(<26)$ $\mathrm{mmHg}$, but his distal contractile integral (DCl) was very high at 21,464 $(1500-13,000) \mathrm{mmHg} \mathrm{s} \mathrm{cm}$. Esophageal peristalsis was preserved. Therefore, the patient was diagnosed as having an epiphrenic esophageal diverticulum derived from a jackhammer esophagus for which laparoscopic transhiatal diverticulectomy and Heller-Dor procedure were performed. The postoperative course was uneventful. His symptoms improved, and the level of DCl also returned to a normal level of $3867 \mathrm{mmHg} \mathrm{s} \mathrm{cm}$ at 2 months after the operation.

Conclusion: Laparoscopic transhiatal diverticulectomy and esophagomyotomy can be useful procedures for an epiphrenic esophageal diverticulum derived from a jackhammer esophagus due to their lower invasiveness.

Keywords: Jackhammer esophagus, Epiphrenic esophageal diverticulum, Myotomy

\section{Background}

Esophageal diverticulum has been reported to occur at a frequency of $1 \%$ among all gastrointestinal diverticula [1]. The epiphrenic esophageal diverticulum, a pulsion diverticulum caused by the pressure of esophageal muscle movement applied to the weak part of the lower

\footnotetext{
* Correspondence: a-yoshimura@oita-u.ac.jp

${ }^{1}$ Department of Gastroenterological and Pediatric Surgery, Oita University

Faculty of Medicine, Idaigaoka 1-1, Hasama-machi, Oita 879-5593, Japan

Full list of author information is available at the end of the article
}

esophagus, is often associated with esophageal motility disorders [2, 3]. In recent years, high-resolution manometry (HRM) has been developed as a manometric method to assess the esophagus. In the new Chicago Classification, the diagnostic criteria for esophageal motility disorder were revised based on HRM techniques.

Jackhammer esophagus is characterized by esophageal hypercontraction and is a rare disorder among the esophageal motility disorders [4-6]. In the 2012 Chicago Classification, jackhammer esophagus was identified as a

\section{Springer Open}

(c) The Author(s). 2020 Open Access This article is licensed under a Creative Commons Attribution 4.0 International License, which permits use, sharing, adaptation, distribution and reproduction in any medium or format, as long as you give appropriate credit to the original author(s) and the source, provide a link to the Creative Commons licence, and indicate if changes were made. The images or other third party material in this article are included in the article's Creative Commons licence, unless indicated otherwise in a credit line to the material. If material is not included in the article's Creative Commons licence and your intended use is not permitted by statutory regulation or exceeds the permitted use, you will need to obtain permission directly from the copyright holder. To view a copy of this licence, visit http://creativecommons.org/licenses/by/4.0/. 
subtype of nutcracker esophagus (NE) [7]. We present a patient who successfully underwent laparoscopic transhiatal surgery for an epiphrenic esophageal diverticulum derived from a jackhammer esophagus diagnosed with HRM.

\section{Case presentation}

A 78-year-old Japanese man presented to the hospital with dysphagia. His symptom remained even after treatment with proton pump inhibitors. Upper gastrointestinal endoscopy found a 31-mm diverticulum on the left wall in the lower part of the esophagus (Fig. 1a). The mucosa of the diverticulum was normal. Moreover, a barium esophagogram showed a diverticulum in the lower esophagus $50 \mathrm{~mm}$ above the esophagogastric junction (EGJ) and no obstruction of contrast at the lower esophageal sphincter (Fig. 1b). Chest computed tomography $(\mathrm{CT})$ also revealed a diverticulum on the left wall in the lower esophagus (Fig. 1c). There was no history of esophageal motility disorder in the patient's family.

Upon admission, his height, weight, and body mass index were $158 \mathrm{~cm}, 66.2 \mathrm{~kg}$, and $26.5 \mathrm{~kg} / \mathrm{m}^{2}$, respectively. Laboratory tests showed no anemia and revealed normal liver and kidney functions. HRM by Starlet (STAR
MEDICAL, Tokyo, Japan) was performed to determine the presence of any esophageal motility disorder. The HRM catheter was inserted transnasally and placed in the optimal position in the esophagus, and the distal sensor was placed 2 to $3 \mathrm{~cm}$ below the diaphragm. A standard HRM protocol consists of a baseline quiescent period lasting at least $30 \mathrm{~s}$, followed by a series of 105 $\mathrm{mL}$ swallows of room temperature water with the patient in the supine or reclined position [8]. The patient's integrated relaxation pressure (IRP) was normal at 25.9 (normal <26) $\mathrm{mmHg}$, whereas his distal contractile integral (DCI) was very high at 21,464 (normal 1500-13, 000) $\mathrm{mmHg} \mathrm{s} \mathrm{cm}$. Esophageal peristalsis was preserved (Fig. 2a). Therefore, the patient was diagnosed as having an epiphrenic esophageal diverticulum derived from a jackhammer esophagus. Then, a laparoscopic diverticulectomy and esophagomyotomy (Heller procedure) were performed.

After taping the abdominal esophagus, we dissected tissues along the esophagus to the oral side. An esophageal diverticulum was observed on the left side of the esophageal wall $5 \mathrm{~cm}$ above the EGJ (Fig. 3b). The diverticulectomy was performed using a linear stapler while confirming the lumen of the esophagus under

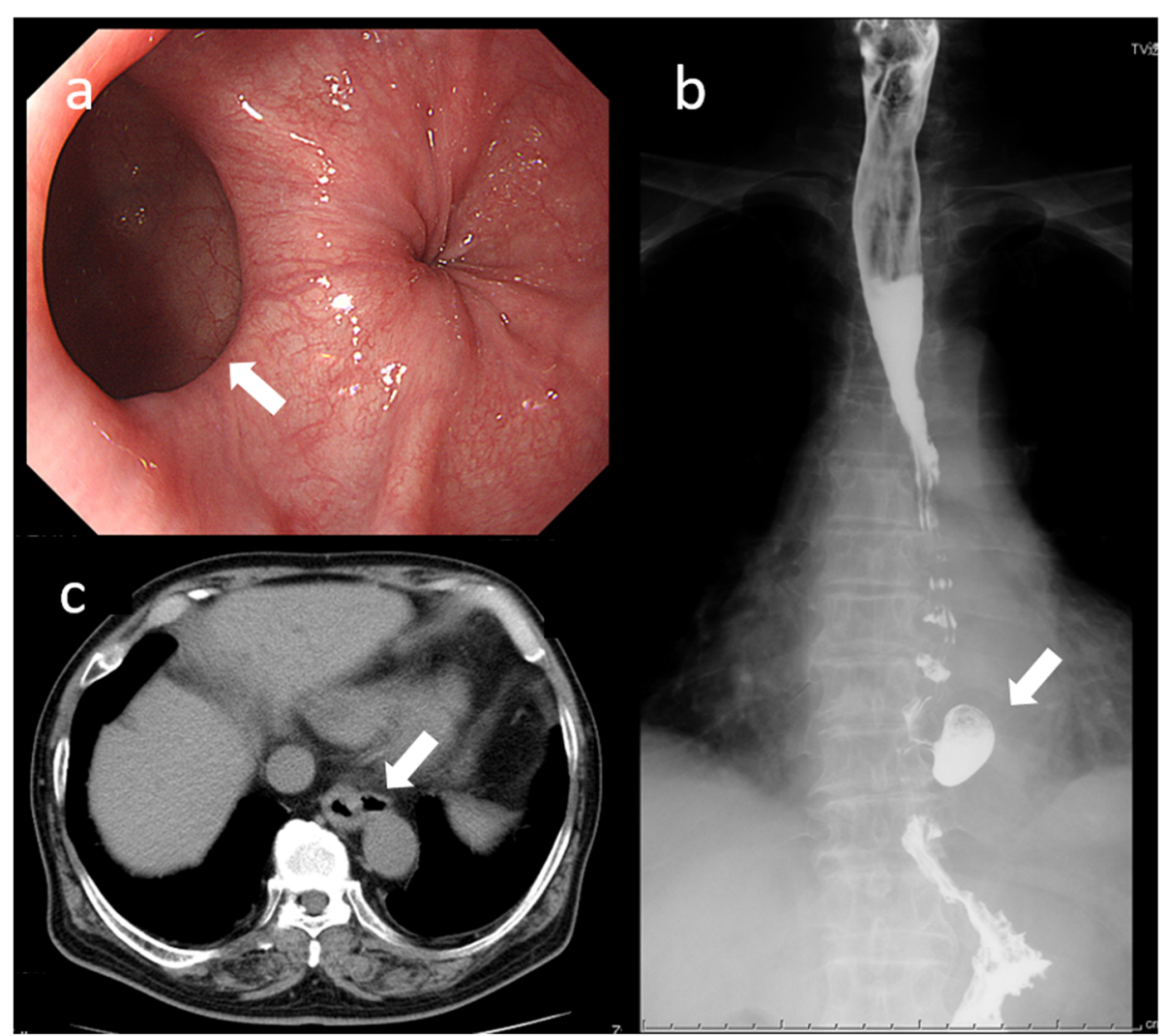

Fig. 1 a Upper gastrointestinal endoscopy showed a 31-mm diverticulum on the left wall of the lower esophagus (arrow). b A barium esophagogram showed a diverticulum in the lower esophagus $50 \mathrm{~mm}$ above the EGJ (arrow). c Chest CT revealed a diverticulum on the left wall in the lower esophagus with no esophageal masses (arrow) 


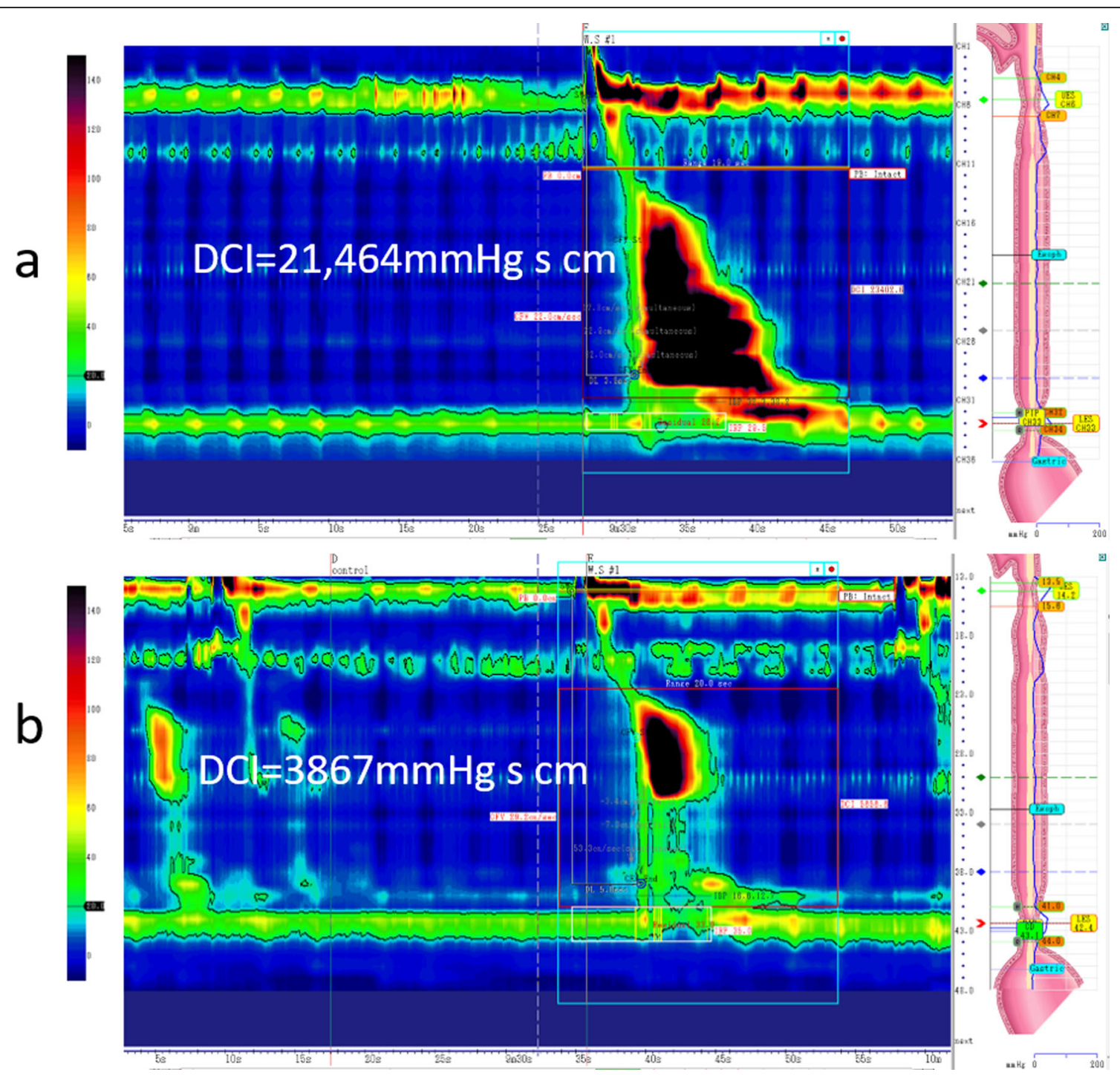

Fig. 2 a Preoperative HRM. DCl was high at $21,464 \mathrm{mmHg} \mathrm{s} \mathrm{cm}$. b Postoperative HRM. The level of DCl improved to normal at $3867 \mathrm{mmHg} \mathrm{s} \mathrm{cm}$

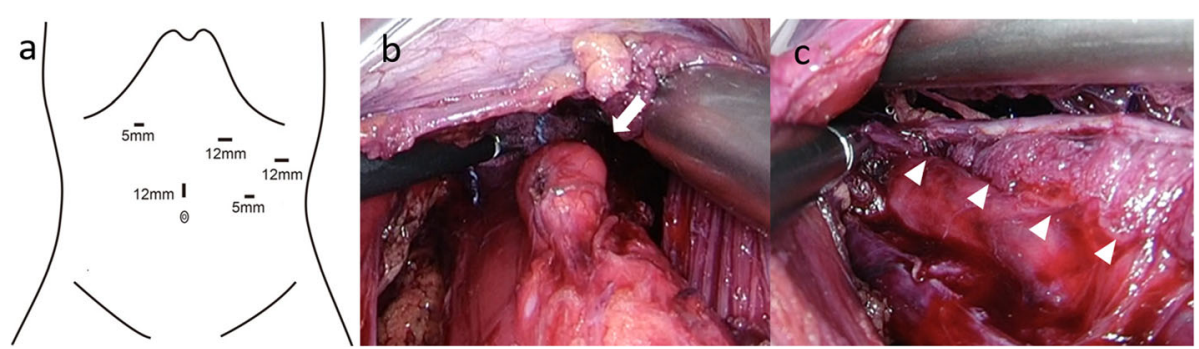

Fig. 3 a Trocar arrangement in this procedure. $\mathbf{b}$ An esophageal diverticulum was observed on the left side of the esophageal wall $5 \mathrm{~cm}$ above the EGJ (arrow). c Esophagomyotomy of the front wall was performed from the EGJ to $10 \mathrm{~cm}$ above the EGJ 
observation with intraoperative esophagoscopy, and the staple line was reinforced. An esophagomyotomy of the front wall was also performed from the EGJ to $10 \mathrm{~cm}$ above the EGJ (Fig. 3c). As the patient's IRP level was normal from the findings of HRM, we did not perform gastric myotomy. Finally, the Dor procedure was performed. The operation time and blood loss were 270 min and $5 \mathrm{~mL}$, respectively. The diverticulum specimen was $30 \mathrm{~mm} \times 20 \mathrm{~mm}$ in diameter with normal mucosa. The diverticulum wall lacked a proper muscular layer of the esophagus. The pathological diagnosis was a pseudodiverticulum with no evidence of malignancy.

The patient's postoperative course was uneventful, and he was discharged from the hospital 12 days after surgery. At 2 months after surgery, his symptoms had improved, and his DCI had returned to a normal level of $3867 \mathrm{mmHg} \mathrm{s} \mathrm{cm} \mathrm{(Fig.} \mathrm{2b).}$

\section{Discussion}

Esophageal diverticulum derived from a jackhammer esophagus is considered to be a very rare disease that is difficult to diagnose. In the present case, from the findings of HRM, we could diagnose the patient as having a jackhammer esophagus. After the laparoscopic transhiatal diverticulectomy and esophagomyotomy, his symptoms and DCI level improved.

In recent years, HRM has been developed as a method of esophageal manometry. HRM uses a high-resolution catheter to transmit intraluminal pressure data that is subsequently converted into dynamic esophageal pressure topography plots [9]. Metric data from these plots are synthesized to yield an esophageal motility diagnosis according to the Chicago Classification version 3.0, a formal analytic scheme for esophageal motility disorders [7]. In the 2012 Chicago Classification, jackhammer esophagus was identified as a subtype of NE and was defined as an esophageal motility disorder characterized by a DCI greater than $8000 \mathrm{mmHg} \mathrm{s} \mathrm{cm} \mathrm{with} \mathrm{normal} \mathrm{IRP}$ in at least two of ten swallows on manometry. Jackhammer esophagus is rare, occurring in only $4.1 \%$ of patients undergoing HRM in a series of 2000 patients [6]. We could make an accurate diagnosis from the findings of HRM, and we thought HRM was useful to diagnose esophageal motility disorders.

Esophageal diverticulum has been reported to occur at a frequency of $1 \%$, the lowest among all gastrointestinal diverticula [1]. The types of esophageal diverticulum include pharyngoesophageal diverticulum (Zenker's diverticulum), middle esophageal diverticulum, and epiphrenic esophageal diverticulum, which occurs at a frequency of $11 \%$ among esophageal diverticula [1]. Epiphrenic esophageal diverticulum is a pulsion diverticulum caused by the pressure of esophageal muscle movement applied to the weak part of the lower esophagus [2]. Surgical treatment should be actively performed for those with severe symptoms such as dysphagia and pain and those with growing or poor drainage of content in the diverticulum [3]. The esophageal diverticulum is often associated with esophageal motility disorders [3]. Khullar et al. have reported favorable results of diverticulectomy and esophagomyotomy for esophageal diverticula derived from jackhammer esophagus [10]. They suggested that surgery is necessary not only for diverticula but also for esophageal motility disorder.

Endoscopic or surgical esophagomyotomy can be performed for esophageal motility disorder. There are only few reports of per-oral endoscopic myotomy (POEM) and surgical myotomy for non-achalasia motility disorders. Hoppo et al. reported that POEM was performed in 5 cases of jackhammer esophagus, 1 case of diffuse esophageal spasm (DES), and 2 cases of NE, with improvement of $75 \%$ for dysphagia and $80 \%$ for chest pain [11]. Schlottmann et al. performed surgical myotomy in 19 cases of DES and 12 cases of NE, of which 15 of the DES (80\%) and 6 of the NE (50\%) cases improved [12]. To the best of our knowledge, there are only two reports of surgical esophagomyotomy performed for jackhammer esophagus, and the results were good [10, 13]. Therefore, esophagomyotomy could be effective for jackhammer esophagus. Rossetti et al. reported that esophagomyotomy can be performed just above the lower esophageal sphincter (LES) in patients with epiphrenic esophageal diverticulum with normal function of the LES $[14,15]$. As the patient's IRP level was normal from the findings of HRM, we did not perform gastric myotomy in this patient.

It is reported that laparoscopic transhiatal manipulation is possible up to the middle esophagus [16]. With the progress in laparoscopic surgery [17], laparoscopic transhiatal esophagomyotomy for esophageal motility disorder even in the superior portion could be performed safely. Conventionally, following esophageal surgery, there are possibilities of postoperative respiratory complications due to the adverse effects of thoracotomy and associated one-lung ventilation [18]. However, a transhiatal procedure without thoracotomy is less invasive and has fewer postoperative complications [19]. There is no consensus of the length of esophagomyotomy in patients with esophageal diverticulum [20]. Therefore, both the findings of HRM and possible manipulations should be considered to determine the length of esophagomyotomy. The extent of the lesions, including the diverticulum, was mainly in the middle and lower esophagus; therefore, we believed that laparoscopic transhiatal diverticulectomy and esophagomyotomy from the EGJ to $10 \mathrm{~cm}$ above the EGJ would improve esophageal diverticulum and motility disorder. Since the location of the diverticulum was typical of the 
left esophageal wall, esophagomyotomy of the front wall was performed safely. After surgery, the patient's DCI level improved from 21,464 to $3867 \mathrm{mmHg} \mathrm{s} \mathrm{cm}$ along with his symptoms. The findings in our patient showed that laparoscopic transhiatal diverticulectomy and esophagomyotomy can be useful procedures.

\section{Conclusions}

Esophageal motility disorder, which should be treated from the lower to the superior portion of the esophagus, could be treated by the laparoscopic transhiatal approach. The laparoscopic procedures of transhiatal diverticulectomy and esophagomyotomy are less invasive and can be useful for treating an epiphrenic esophageal diverticulum derived from a jackhammer esophagus.

\begin{abstract}
Abbreviations
HRM: High-resolution manometry; NE: Nutcracker esophagus;

EGJ: Esophagogastric junction; CT: Computed tomography; IRP: Integrated relaxation pressure; DCl: Distal contractile integral; POEM: Per-oral endoscopic myotomy; DES: Diffuse esophageal spasm; LES: Lower esophageal sphincter
\end{abstract}

\section{Acknowledgements}

The authors would like to thank Rise Japan, LCC (http://rise-japan.rulez.jp/) for the English language review.

\section{Authors' contributions}

$A F, T S, T E, K T, K S, K N, K O, Y K, T H, T A, Y U, M T, H S$, and NS drafted the manuscript. MI gave the final approval of the version to be published. All authors read and approved the final manuscript.

\section{Funding}

This study was not funded by any grants.

\section{Availability of data and materials}

The data are not available for public access because of patient privacy concerns but are available from the corresponding author on reasonable request.

\section{Ethics approval and consent to participate}

The publication of this case report was approved by the local institutional ethics committee.

\section{Consent for publication}

The case report and publication process were explained to the patient, and he granted permission to publish the report.

\section{Competing interests}

The authors declare that they have no competing interests.

\section{Author details}

${ }^{1}$ Department of Gastroenterological and Pediatric Surgery, Oita University Faculty of Medicine, Idaigaoka 1-1, Hasama-machi, Oita 879-5593, Japan. ${ }^{2}$ Department of Gastroenterological Surgery, Oita Nakamura Hospital, Oita, Japan. ${ }^{3}$ Department of Comprehensive Surgery for Community Medicine, Oita University Faculty of Medicine, Oita, Japan.

Received: 5 March 2020 Accepted: 9 June 2020

Published online: 29 June 2020

\section{References}

1. Postlethwait RW. Diverticula of the esophagus. In: Postlethwait RW, editor. Surgery of the esophagus. 2nd ed. Norwalk: Appleton Century Crofts; 1986. p. 129-59.

2. Cross F, Johnson G, Gerein A. Esophageal diverticula: associated neuromuscular changes in the esophagus. Arch Surg. 1961;83:525-33.
3. Debas HT, Payne WS, Cameron AJ, Carlson HC. Physiopathology of lower esophageal diverticulum and its implications for treatment. Surg Gynecol Obstet. 1980;151:593-600.

4. Roman S, Tutuian R. Esophageal hypertensive peristaltic disorders. Neurogastroenterol Motil. 2012;24(Suppl 1):32-9.

5. Tsuboi K, Mittal SK, Legner A, Yano F, Filipi CJ. Relationship between manometric findings and reported symptoms in nutcracker esophagus: insights gained from a review of 313 patients. J Gastroenterol. 2010;45: 1033-8.

6. Roman S, Pandolfino JE, Chen J, Boris L, Luger D, Kahrilas PJ. Phenotypes and clinical context of hypercontractility in high-resolution esophageal pressure topography (EPT). Am J Gastroenterol. 2012;107:37-45.

7. Bredenoord AJ, Fox M, Kahrilas PJ, Pandolfino JE, Schwizer W, Smout AJ International High Resolution Manometry Working Group. Chicago Classification criteria of esophageal motility disorders defined in high resolution esophageal pressure topography. Neurogastroenterol Motil. 2012: 24 Suppl 1:57-65.

8. Patel A, Ding A, Mirza F, Gyawali CP. Optimizing the high-resolution manometry (HRM) study protocol. Neurogastroenterol Motil. 2015;27:300-4.

9. Yadlapati R. High-resolution esophageal manometry: interpretation in clinical practice. Curr Opin Gastroenterol. 2017:33:301-9.

10. Khullar OV, Shroff SR, Sakaria SS, Force SD. Midesophageal pulsion diverticulum resulting from hypercontractile (jackhammer) esophagus. Ann Thorac Surg. 2017;103:e127-9.

11. Hoppo T, Thakkar SJ, Schumacher LY, Komatsu Y, Choe S, Shetty A, et al. A utility of peroral endoscopic myotomy (POEM) across the spectrum of esophageal motility disorders. Surg Endosc. 2016;30:233-44.

12. Schlottmann F, Shaheen NJ, Madanick RD, Patti MG. The role of Heller myotomy and POEM for nonachalasia motility disorders. Dis Esophagus. 2017;30:1-5.

13. Vázquez García P, Ciriza de Los Ríos C, Canga Rodríguez-Valcárcel F, Hernández García-Gallardo D. Progression of jackhammer esophagus to type III achalasia and improvement after extended myotomy. J Neurogastroenterol Motil. 2020;26:164-6.

14. Rossetti G, Fei L, del Genio G, Maffettone V, Brusciano L, Tolone S, et al. Epiphrenic diverticula mini-invasive surgery: a challenge for expert surgeons-personal experience and review of the literature. Scand I Surg. 2013;102:129-35.

15. Macke RA, Luketich JD, Pennathur A, Bianco V, Awais O, Gooding WE, et al. Thoracic esophageal diverticula: a 15-year experience of minimally invasive surgical management. Ann Thorac Surg. 2015;100:1795-802.

16. Shiozaki A, Fujiwara H, Konishi H, Morimura R, Komatsu S, Murayama Y, et al. Middle and lower esophagectomy preceded by hand-assisted laparoscopic transhiatal approach for distal esophageal cancer. Mol Clin Oncol. 2014;2: $31-7$

17. Inomata M, Shiroshita H, Uchida H, Bandoh T, Akira S, Yamaguchi S, et al. Current status of endoscopic surgery in Japan: The 14th National Survey of Endoscopic Surgery by the Japan Society for Endoscopic Surgery. Asian J Endosc Surg. 2020;13:7-18.

18. Zingg U, Forberger J, Frey DM, Esterman AJ, Oertli D, Beck-Schimmer B, et al. Inflammatory response in ventilated left and collapsed right lungs, serum and pleural fluid, in transthoracic esophagectomy for cancer. Eur Cytokine Netw. 2010;21:50-7.

19. Mori K, Aikou S, Yagi K, Nishida M, Mitsui T, Yamagata Y, et al. Technical details of video-assisted transcervical mediastinal dissection for esophageal cancer and its perioperative outcome. Ann Gastroenterol Surg. 2017;1:232-7.

20. Rosati R, Fumagalli U, Elmore U, de Pascale S, Massaron S, Peracchia A Long-term results of minimally invasive surgery for symptomatic epiphrenic diverticulum. Am J Surg. 2011;201:132-5.

\section{Publisher's Note}

Springer Nature remains neutral with regard to jurisdictional claims in published maps and institutional affiliations. 